\title{
Evaluación de la protección del estátor de generadores sincrónicos ante fallas a tierra por medio del método de inyección subarmónica
}

\author{
Luis C. Giraldo*, Juan J. Mora-Flórez**\$, Gabriel Olguin*** \\ * Ingeniería, ABB Transformadores, Pereira, Colombia \\ ** Programa de Ingeniería Eléctrica, Universidad Tecnológica de Pereira, Pereira, Colombia \\ *** Corporate Research, ABB, Vasteras, Suecia \\ §e-mail:jjmora@utp.edu.co
}

(Recibido: Agosto 23 de 2007 - Aceptado: Abril 4 de 2008)

\begin{abstract}
Resumen
En este artículo se hace una evaluación de la protección completa del estátor de generadores sincrónicos ante fallas a tierra utilizando el método de inyección subarmónica. Primeramente, se hace una descripción de dos enfoques para la protección completa del estátor: el método del tercer armónico y el método de inyección subarmónica. Después, se lleva a cabo un análisis del error en la determinación de la resistencia de falla a tierra para un generador sincrónico de $11.4 \mathrm{kV}$, considerando diversas condiciones de operación. Este error está relacionado con el tipo de transformadores de voltaje utilizados, la presencia de ruido en las señales, el proceso de digitalización y las variaciones en los valores numéricos de los parámetros requeridos. Finalmente, se demuestra que el método de inyección subarmónica es muy útil en la determinación de la resistencia de falla a tierra y que tiene un buen desempeño en la protección completa del estátor.
\end{abstract}

Palabras clave: Resistencia de falla, Generador sincrónico, Método de inyección subarmónica, Método del tercer armónico, Protección completa del estátor.

ELECTRICAL ENGINEERING

\section{Evaluation of the stator ground-fault protection for synchronous generators by means of the subharmonic injection method}

\begin{abstract}
In this paper, we make an evaluation of the complete ground-fault protection of the stator of synchronous generators by use of the subharmonic injection method. Firstly, we describe two approaches for the complete protection of the stator: the third harmonic method and the method of subharmonic injection. Secondly, we analyze the error in the determination of the ground-fault resistance for an $11.4 \mathrm{kV}$ synchronous generator from the consideration of various operating conditions. This error is related to the type of voltage transformers being used, the occurrence of signal noise, the digitalization process, and variations in the numerical values of the required parameters. Lastly, we show that the subharmonic injection method is very useful for the determination of the ground-fault resistance and performs well for the complete protection of the stator.
\end{abstract}

Keywords: Ground-fault resistance, Synchronous generator, Subharmonic injection method, Third harmonic method, Stator ground-fault protection. 


\section{Introducción}

Los sistemas eléctricos de potencia han tenido una gran evolución en los últimos años, debido al crecimiento de la población y la necesidad de brindar un servicio eficiente y de alta calidad. A partir de la implementación de los nuevos esquemas de regulación del sector eléctrico a nivel mundial, el problema de la calidad de la prestación del servicio de energía eléctrica se ha convertido en foco de especial interés para las empresas generadoras, transmisoras y operadoras de red. Algunas de las normas establecidas surgen de la necesidad de buscar soluciones a las interrupciones, de modo que las empresas que suministran energía eléctrica aseguren continuidad en el servicio, garantizando una plena confiabilidad en todos los equipos involucrados en el sistema de potencia (Reimert, 2006).

Las fallas de fase a tierra en el estátor de un generador sincrónico son en gran medida el tipo de anomalías más comunes que se presentan en estas máquinas y una de las principales causas de su salida de servicio (Reimert, 2006; IEEE,1995).

Una primera falla de fase a tierra en el neutro de un generador (puesto a tierra mediante una impedancia de aterrizaje), no es perjudicial para la máquina; sin embargo, ésta puede conducir a una alta corriente de falla si una segunda falla a tierra ocurre, lo que implica un daño severo en el núcleo del estátor y pérdidas asociadas a la energía no suministrada por la parada de las máquinas en la central de generación. Por lo anterior, se hace necesario identificar rápidamente la presencia de una falla a tierra en cualquier parte del devanado (IEEE,1985).

La protección convencional para la detección de la falla a tierra del estátor sólo proporciona protección sensible para cerca del $90 \%$ al $95 \%$ del estátor, medido desde terminales hacia el neutro (ANSI / IEEE,1989). Esto es debido a que la falla en el $10 \%$ o $5 \%$ restante del devanado, cerca del neutro, no origina suficiente tensión y corriente residual a frecuencia fundamental para causar la operación de estos relés (Giraldo et al., 2006; Powell, 1998; García \& Alcántara, 2006). Debido a lo anterior, es necesario implementar opciones alternativas de protección $100 \%$, que garanticen la total protección de los devanados de la máquina (Illar et al., 1979; Wu \& Lu, 2002).

En este artículo, se presenta un análisis de los errores del método de inyección subarmónica, que se utiliza como protección del $100 \%$ del estátor de generadores sincrónicos ante fallas de fase a tierra. Los errores considerados son aquellos debidos a los equipos de transformación, a la presencia de ruido en las señales y al proceso de digitalización.

En la Sección 2 se presentan el principio de operación y las limitaciones de las protecciones convencionales de falla a tierra. En la Sección 3 se describe y analiza detalladamente el método de protección $100 \%$ basado en inyección subarmónica. El modelado del sistema prototipo, las diferentes pruebas y los resultados más representativos del método que conllevan al análisis de error producido en la estimación de la resistencia de falla, se muestran en la Sección 4. El análisis de los resultados se realiza en la Sección 5. Finalmente, las principales conclusiones de la investigación se presentan en la Sección 6.

\section{Protección convencional ante fallas de fase a tierra}

El esquema de protección utilizado en generadores puestos a tierra a través de una impedancia, consiste en un relé de sobretensión con retardo de tiempo (59 GN), conectado para medir tensión de secuencia cero, tal como se muestra en la Figura 1.

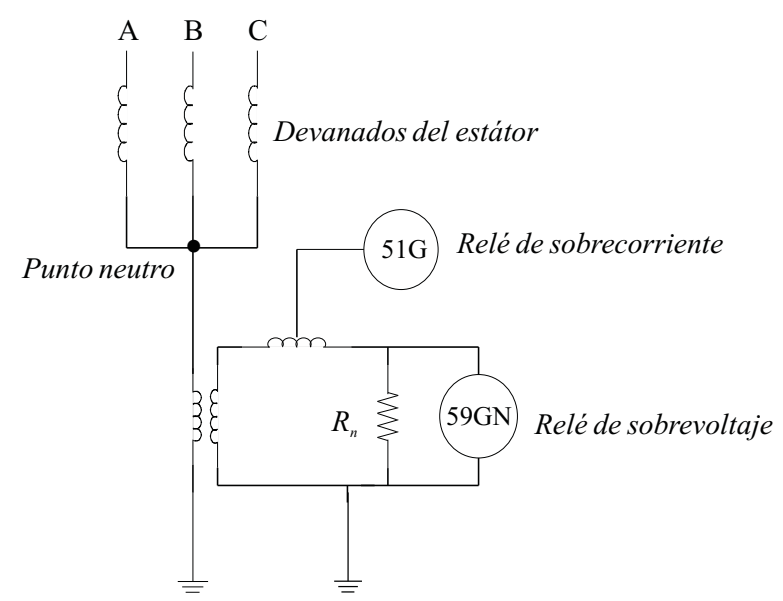

Figura 1. Esquema de protección convencional ante fallas a tierra del generador sincrónico. 
La tensión de secuencia cero es máxima para una falla en terminales y disminuye 'linealmente' a medida que la falla se presenta más cerca del neutro. Así, la tensión o corriente de falla es proporcional al número de espiras en corto circuito, tal como se ilustra en la Figura 2 para un generador de $11.4 \mathrm{kV}$ (utilizado como prototipo de prueba en la investigación aquí presentada). La figura también muestra el efecto de variación de la resistencia de falla en la tensión de secuencia cero, durante situaciones de falla a tierra (Fulczyk, 2001).

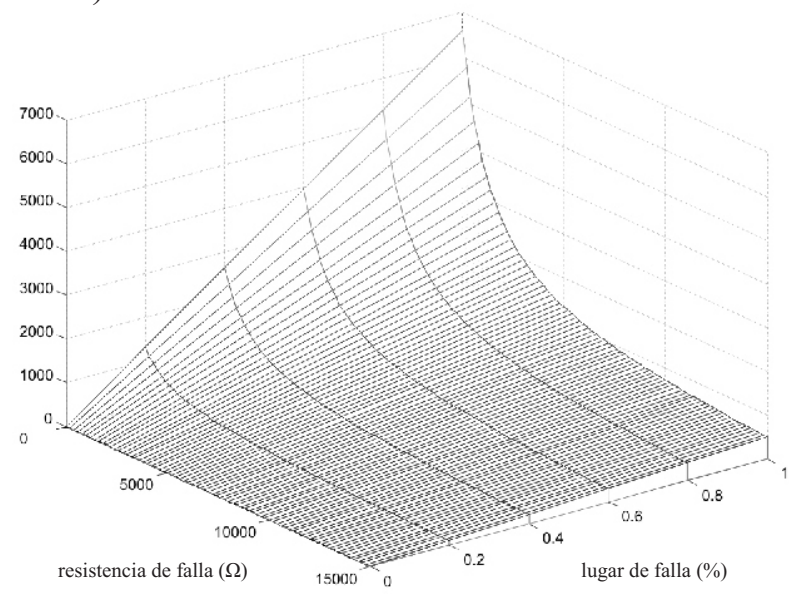

Figura 2. Tensión de secuencia cero en el generador durante situaciones de falla a tierra a lo largo de los enrollados de la máquina.

En general, las protecciones 'convencionales' contra falla a tierra miden la corriente o tensión de secuencia cero y actúan cuando se sobrepasa un nivel de ajuste determinado, lo cual únicamente funciona bien para fallas más allá del $10 \%$ del devanado (medido desde el punto neutro).

\section{Protección $\mathbf{1 0 0} \%$ del estátor ante fallas de fase a tierra}

Para proteger la totalidad del devanado del estátor, los métodos más comúnmente utilizados son el método del tercer armónico y el método de inyección subarmónica (Reimert, 2006; Allen \&Waldorf, 1946; Dann \& Montsinger, 1934). El método del tercer armónico utiliza estas componentes medidas en las terminales y en el punto neutro de las máquinas $\mathrm{y}$, mediante su comparación, garantiza protección total a los devanados.
El método de inyección de tensión detecta fallas a tierra aplicando una tensión entre el neutro y la tierra, a una frecuencia subarmónica determinada. La magnitud medida es la corriente resultante de la correspondiente tensión inyectada. Cuando ocurre una falla de fase a tierra, la corriente resultante varía incrementando su valor, lo que causa la actuación de las protecciones.

Existen dos esquemas de inyección subarmónica para proteger el 100\% del estátor (Giraldo et al., 2006): (a) inyección de una señal en paralelo a la resistencia de puesta a tierra de la máquina, y (b) inyección de señal en serie con una parte de la resistencia puesta a tierra de la máquina.

En este artículo, se presenta un análisis del esquema basado en la inyección en serie a la resistencia de puesta a tierra del generador, tal como se muestra en la Figura 3. Para este caso, la fuente de inyección (E) se conecta al lado secundario del transformador de aterrizaje $(\mathrm{T})$, mediante un filtro (F), que permite sólo el paso de la correspondiente frecuencia de inyección y bloquea la señal fundamental de secuencia. Durante la operación normal de la máquina, hay una pequeña corriente de carga circulando a la frecuencia de inyección. Cuando ocurre una falla, la admitancia a tierra de la máquina aumenta y la corriente se incrementa causando una alarma o disparo de la protección (Allen \& Waldorf, 1946).

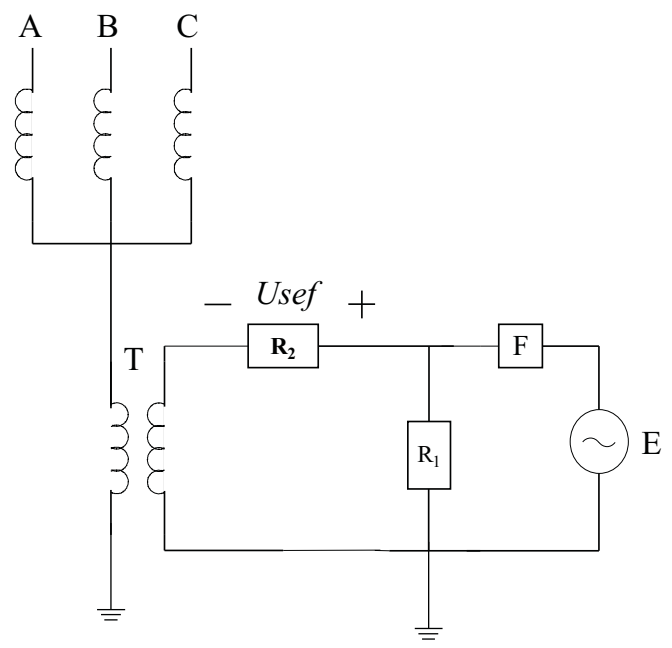

Figura 3. Esquema de inyección en serie a la resistencia de puesta a tierra. 
Este esquema de inyección divide la resistencia de puesta a tierra en dos resistencias $\left(R_{1}\right.$ y $\left.R_{2}\right)$, normalmente con una relación 4.5:1. En la resistencia de menor valor $\left(\mathrm{R}_{1}\right)$ se inyecta la señal y en la resistencia de mayor valor $\left(\mathrm{R}_{2}\right)$, se mide la tensión correspondiente a la frecuencia de inyección (normalmente $1 / 4$ de la frecuencia fundamental).

A partir del esquema circuital mostrado en la Figura 3, se puede obtener la siguiente expresión que sirve para determinar la sensibilidad del esquema de inyección.

$$
R_{f}^{\prime} \leq \frac{\left(X_{c}^{\prime}\right)^{2}\left(R_{1}+R_{i}\right)}{2\left[R_{i}\left(R_{1}+R_{2}\right)+R_{1} R_{2}\right]}
$$

Esta expresión representa la máxima resistencia de falla que se puede detectar.

La sensibilidad del esquema de inyección está determinada por los parámetros del generador y de la unidad de inyección. $X_{c}$ es la reactancia capacitiva a tierra del generador sincrónico a la frecuencia fundamental.

Este esquema permite detectar fallas cerca del punto neutro y estimar la resistencia de falla mediante la medida de tensión $\left(U_{\text {sef }}\right)$. El objeto de esta investigación es estimar el error en el cálculo de la resistencia de falla, considerando diferentes condiciones de operación del generador.

\section{Pruebas y resultados}

El error en la determinación de la resistencia de falla se debe básicamente a las siguientes consideraciones: a) los transformadores de voltaje (Pts) y transformadores de corriente (Cts) tienen una clase de precisión, lo que conduce a variaciones del valor real medido dentro de un rango de incertidumbre.

b) en la práctica, la máquina está sujeta a perturbaciones externas que se manifiestan como interferencia y/o ruido. Debido a esto, la señal subarmónica monitoreada entre el neutro y la tierra no es exactamente la inyectada.

c) en el proceso de digitalización, la cuantización de la señal involucra errores en las medidas de tensión introducidos a partir de la conversión análogo / digital, determinada por la resolución del convertidor utilizado.

d) debido a efectos del tiempo, temperatura (entre otras) existen variaciones en el valor numérico de algunos parámetros alrededor de su valor real.

Considerando los aspectos anteriormente mencionados, se propone el análisis del método de inyección subarmónica. Los parámetros de la máquina de prueba utilizada y las pruebas realizadas, se presentan en esta sección. Las pruebas permiten hallar el máximo error presente en la estimación de la resistencia de falla, bajo diferentes condiciones de operación de la máquina.

\subsection{Parámetros del generador y de los métodos de protección}

El generador utilizado como prototipo de prueba se presenta en la Tabla 1 (García \& Alcántara, 2006).

Tabla.1. Parámetros del generador prototipo utilizado en las pruebas.

\begin{tabular}{lc}
\hline \multicolumn{1}{c}{ Parámetro considerado } & Valor nominal \\
\hline Potencia nominal $\left(S_{N}\right)$ & $850 \mathrm{MVA}$ \\
Velocidad síncrona $(W)$ & $3,000 \mathrm{rpm}$ \\
Frecuencia de operación $\left(f_{o}\right)$ & $50 \mathrm{~Hz}$ \\
Tensión nominal $\left(U_{n}\right)$ & $11.4 \mathrm{kV}$ \\
Resistencia de puesta a tierra $\left(R_{n}\right)$ & $2,054 \mathrm{k} \Omega$ \\
Capacitancia a tierra del estátor del generador $\left(C_{g}\right)$ & $0.385 \mu \mathrm{F} /$ fase \\
Resistencia de aislamiento & $10 \mathrm{M} \Omega$ \\
\hline
\end{tabular}


Tabla. 2. Parámetros de la unidad de inyección del esquema de inyección subarmónico.

\begin{tabular}{lc}
\hline \multicolumn{1}{c}{ Parámetro } & Valor numérico \\
\hline Magnitud tensión de inyección $(E)$ & $50 \mathrm{~V}$ \\
Resistencia interna de la fuente $\left(R_{i}\right)$ & $8 \Omega$ \\
Frecuencia de operación de inyección $(f)$ & $12.5 \mathrm{~Hz}$ \\
\hline
\end{tabular}

Tabla 3. Valores nominales del transformador lineal de aterrizaje.

\begin{tabular}{lc}
\hline \multicolumn{1}{c}{ Parámetro considerado } & Valor nominal \\
\hline Capacidad nominal & $25 \mathrm{kVA}$ \\
Frecuencia nominal & $50 \mathrm{~Hz}$ \\
Pérdidas en el núcleo & $100 \mathrm{~W}$ \\
Pérdidas en el cobre & $290 \mathrm{~W}$ \\
Tensión de cortocircuito (\% de la tensión nominal) & 3 \\
Corriente de excitación (\% de la corriente nominal) & 2 \\
Relación de transformación & $11,400 \mathrm{kV} / 240 \mathrm{~V}$ \\
\hline
\end{tabular}

Los parámetros de la unidad de inyección del esquema de protección del $100 \%$ se presentan en la Tabla 2. Los parámetros del transformador de aterrizaje se muestran en la Tabla 3. Este transformador se considera lineal, debido a que bajo condición de falla no se satura, característica propia de los transformadores de puesta a tierra.

Para la realización de las diferentes pruebas se utilizó el software Matlab-Simulink ${ }^{\circledR}$.

\subsection{Determinación numérica de la resistencia de falla}

Para el esquema de inyección, las medidas disponibles son la magnitud y el ángulo de la tensión en la resistencia de puesta a tierra $\left(\mathrm{R}_{2}\right)$, tal como se presenta en la Figura 3. A partir de la medida de tensión $\left(U_{\text {sef }}\right)$ y de los parámetros del modelo circuital, es posible determinar la admitancia compleja de la máquina mediante la siguiente expresión:

$$
Y=\frac{U_{s e f}\left(R_{1}+R_{i}\right)}{\left(R_{1} R_{2} E\right)-U_{s e f}\left[R_{i}\left(R_{1}+R_{2}\right)+R_{1} R_{2}\right]}
$$

De la Ec. (2), referida al lado de alta, se obtiene la expresión para la determinación de la resistencia de falla, así:

$$
R_{k}=\left[\frac{1}{\operatorname{Re}(Y)}\right] N^{2}
$$

donde $R_{k}$ representa la parte real de la admitancia de la máquina, conformada por la resistencia de aislamiento y la resistencia de falla, cuando se considera el transformador de aterrizaje lineal.

\subsection{Incertidumbres en los medidores}

Debido a que la clase de precisión de los medidores eléctricos hace que el valor estimado se encuentre en una tolerancia confiable, entonces se analizan también los posibles errores en la estimación de la resistencia de falla. En esta prueba se considera el medidor con una clase de precisión del 5\%.

Para realizar esta prueba, se varía el valor real de la resistencia de falla obtenida en la medición, dentro del intervalo correspondiente a la incertidumbre del medidor $(5 \%)$. Se realizan varias pruebas con valores dentro del intervalo de cada medidor, 
obteniendo así diferentes valores de resistencia de falla y distintos valores de error relativo, como se muestra en la Tabla 4 en la cual se observa que el error relativo en la medición de la resistencia disminuye con el aumento del valor de la resistencia de falla. Adicionalmente, para variaciones del $5 \%$ del medidor de tensión, se detecta una resistencia de falla con un error máximo del $11 \%$.

\subsection{Presencia de ruido blanco en la señal de inyección}

Las máquinas y los medidores están sujetos a perturbaciones externas que son traducidas como ruido. Sus causas son chisporroteo de escobillas, corrientes desbalanceadas, vibraciones, armónicos, entre otros. Los valores calculados para la resistencia de falla considerando la presencia de ruido blanco, se presentan en las Tablas 5 y 6. Para considerar este efecto, se utiliza la herramienta de software Simulink ${ }^{\circledR} A W G N$ (Add White Gauss Noise), que permite introducir ruido blanco o gaussiano a una señal determinada. A partir de la potencia de la señal de entrada al sistema y la relación señal / ruido, es posible determinar los errores presentes en la estimación de la resistencia de falla, considerando la presencia de ruido blanco en la señal de inyección.

Tabla 4. Error máximo relativo ante una variación del $5 \%$ en el medidor de tensión.

\begin{tabular}{cc}
\hline Resistencia de falla real $(\Omega)$ & Error máximo relativo (\%) \\
\hline 6,000 & 7.36 \\
10,000 & 6.66 \\
14,000 & 6.36 \\
18,000 & 6.21 \\
22,000 & 6.11 \\
26,000 & 6.06 \\
\hline
\end{tabular}

Tabla 5. Error máximo relativo ante un $1 \%$ de ruido en la señal de inyección.

\begin{tabular}{ccc}
\hline Resistencia de falla $(\Omega)$ & Resistencia de falla hallada $(\Omega)$ & Error máximo relativo $(\%)$ \\
\hline 2,000 & 2,008 & 0.31 \\
6,000 & 6,024 & 0.39 \\
10,000 & 10,047 & 0.47 \\
14,000 & 14,078 & 0.56 \\
18,000 & 18,116 & 0.64 \\
22,000 & 22,163 & 0.74 \\
26,000 & 26,218 & 0.84 \\
\hline
\end{tabular}

Tabla 6. Error máximo relativo ante un $10 \%$ de ruido en la señal de inyección.

\begin{tabular}{ccc}
\hline Resistencia de falla $(\Omega)$ & Resistencia de falla hallada $(\Omega)$ & Error máximo relativo $(\%)$ \\
\hline 2,000 & 2,033 & 1.66 \\
6,000 & 6,109 & 1.81 \\
10,000 & 10,227 & 2.27 \\
14,000 & 14,390 & 2.79 \\
18,000 & 18,598 & 3.32 \\
22,000 & 22,852 & 3.87 \\
26,000 & 27,152 & 4.43 \\
\hline
\end{tabular}


Se puede demostrar que la potencia promedio está dada por:

$$
P=\frac{V_{m}^{2}}{2}
$$

donde $V_{m}$ es la magnitud de la tensión $(30 \mathrm{~V}$, aproximadamente).

En la Tabla 5 se observa que el error introducido en la estimación de la resistencia de falla, debido a la presencia del $1 \%$ de ruido en la señal de inyección es menor al $1 \%$.

De la Tabla 6 se aprecia que el error máximo en la estimación de la resistencia, utilizando el esquema de inyección en serie es de alrededor del $4.5 \%$.

\subsection{Errores por cuantización de la señal}

Para fallas de alta impedancia (mayores a $15 \mathrm{k} \Omega$ ), la tensión medida a la frecuencia subarmónica, no presenta una variación significativa, lo que permite que en la práctica no se detecte fácilmente este tipo de fallas. La exactitud de las mediciones depende en gran medida del tratamiento de las señales y sobre todo de la resolución del convertidor análogo-digital.

Esta prueba se realiza para analizar las implicaciones en la asignación de un valor discreto a una señal continua, dependiendo de la resolución del número de bits del convertidor análogo-digital.

Los errores introducidos en la estimación de la resistencia de falla debido al proceso de digitalización de las señales, se presentan en las Tablas 7 y 8 para diferentes resoluciones de los convertidores análogo-digital (ADC).

La resolución de un $\mathrm{ADC}$ está definida como la menor variación que puede ocurrir en la salida digital, como resultado de un cambio en la entrada analógica. La resolución siempre es igual al bit menos significativo (LSB), que se conoce como tamaño de etapa. La resolución está definida por:

$$
R_{o}=\frac{V_{r e f}}{2^{n}}
$$

donde $\left(V_{\text {ref }}\right)$ es el voltaje de referencia de la tarjeta empleada y $n$ es el número de bits del convertidor.

Tabla 7. Error máximo relativo utilizando un conversor de resolución de 8 bits.

\begin{tabular}{ccc}
\hline Resistencia de falla $(\Omega)$ & Resistencia de falla hallada $(\Omega)$ & Error máximo relativo $(\%)$ \\
\hline 2,000 & 1,980 & 0.99 \\
6,000 & 5,960 & 0.67 \\
10,000 & 9,601 & 3.99 \\
14,000 & 13,331 & 4.78 \\
18,000 & 19,418 & 7.88 \\
22,000 & 20,703 & 5.90 \\
26,000 & 26,711 & 2.74 \\
\hline
\end{tabular}

Tabla 8. Error máximo relativo utilizando un conversor de resolución de 12 bits.

\begin{tabular}{ccc}
\hline Resistencia de falla $(\Omega)$ & Resistencia de falla hallada $(\Omega)$ & Error máximo relativo $(\%)$ \\
\hline 2,000 & 1,996 & 0.21 \\
6,000 & 6,010 & 0.16 \\
10,000 & 9,985 & 0.14 \\
14,000 & 14,043 & 0.31 \\
18,000 & 18,083 & 0.46 \\
22,000 & 22,026 & 0.12 \\
26,000 & 26,159 & 0.61 \\
\hline
\end{tabular}


Para realizar las diferentes pruebas, se utilizó la herramienta Cuantizer de Simulink. Utilizando un rango de tarjeta de $10 \mathrm{~V}$ y variaciones en la resolución del ADC de 8 y 12 bits, se obtiene que la salida cuantizada $Y$ en función de la entrada $X$, está dada por:

$$
Y=R_{o} \text { round }\left(\frac{X}{R_{o}}\right)
$$

El intervalo de cuantización de la herramienta Cuantizer está determinado por la Ec. (6), donde $R_{o}$ es la resolución dada por la Ec.(5). Este elemento cuantizador permite discretizar la señal de tensión.

En la Tabla 7 se observa que si se utiliza un ADC con una resolución de 8 bits, el margen de error en la estimación de la resistencia de falla puede ser hasta del $8 \%$.

La Tabla 8 permite observar que para una resolución de 12 bits en el convertidor análogodigital, se pueden presentar errores menores al $1 \%$ del valor real estimado de la resistencia de falla.

\subsection{Errores por variación de la resistencia de aislamiento de la máquina}

Para la realización de esta prueba se considera la variación en el valor de la resistencia de aislamiento, la cual cambia su valor por efecto del tiempo y la temperatura, principalmente.

Para probar el efecto del valor nominal de la resistencia de aislamiento de la máquina, ésta se varía dentro de un intervalo fijo de $10 \mathrm{M} \Omega \pm 50 \%$. De esta forma, se obtienen distintos valores de resistencia de falla y, por consiguiente, errores relativos a partir de variaciones en el valor real de la variable considerada.

En la Tabla 9 se puede apreciar que en la determinación de la resistencia de falla, los errores aumentan con el incremento del valor de la resistencia de la falla. Los errores son menores al $0.3 \%$, permitiendo una muy buena estimación de la resistencia de falla, cuando se presentan variaciones del $50 \%$ en el aislamiento de la máquina.

\section{Análisis de resultados}

El esquema de inyección permite estimar valores grandes de resistencias de falla con una buena exactitud, es decir que el error aumenta para disminuciones en el valor de la resistencia de falla (ver Tabla 4).

El porcentaje de ruido en la señal de inyección determina la exactitud en la estimación de la resistencia de falla. De igual forma, es recomendable disminuir la presencia de ruido mediante filtros que garanticen estimaciones confiables (ver Tablas 5 y 6 ).

La resolución del convertidor análogo-digital empleado, determina la máxima resistencia de falla que puede detectar el esquema de inyección. Por lo tanto, es importante usar resoluciones mayores a los 12 bits, que garanticen la detección de cambios en el valor de la resistencia de falla para alta impedancia (ver Tablas 7 y 8 ).

Las variaciones del valor de la resistencia de aislamiento de la máquina, debidas al envejecimiento, no inciden en gran medida en la precisión de la estimación en la resistencia de falla (Ver Tabla 9).

Tabla 9. Error máximo relativo con variación del $50 \%$ en la resistencia de aislamiento de la máquina.

\begin{tabular}{cc}
\hline Resistencia de falla real $(\Omega)$ & Error máximo relativo $(\%)$ \\
\hline 2,000 & 0.02 \\
6,000 & 0.06 \\
10,000 & 0.10 \\
14,000 & 0.14 \\
18,000 & 0.18 \\
22,000 & 0.22 \\
26,000 & 0.26 \\
\hline
\end{tabular}




\section{Conclusiones}

La protección del $100 \%$ de los devanados del estátor del generador es fundamental para garantizar la confiabilidad de las centrales eléctricas. Los métodos de protección del 100\% del devanado del estátor requieren adicionalmente la protección convencional, para garantizar que mediante la yuxtaposición de las protecciones se proteja efectivamente la totalidad del devanado.

El método de inyección subarmónica provee una adecuada protección de respaldo al generador, inclusive para etapas de parada y de mantenimiento. Esto último se debe a que la unidad de inyección es independiente de la condición de operación en la que se encuentre la máquina.

La estimación numérica de la resistencia de falla es un aspecto importante dentro del método de inyección, debido a que este valor muestra un indicativo del estado de aislamiento de la máquina.

Finalmente, a partir de las pruebas realizadas, se determinó que es necesario utilizar filtros que garanticen la reducción o eliminación de ruido presente en la señal, para garantizar la eliminación de posibles errores en la estimación de la resistencia de falla.

\section{Referencias bibliográficas}

Allen, J. E., \& Waldorf, S. K. (1946). Arcing ground tests on a normally ungrounded $13 \mathrm{kV}$ 3-phase bus. AIEE Transactions on Power Apparatus and Systems 65, 298-306.

ANSI / IEEE (American National Standards Institute / Institute of Electrical and Electronics Engineers). (1989). Standard C62-92. Guide for the application of neutral grounding in electrical utility systems. Part II-Grounding of synchronous generator systems. The Institute of Electrical and Electronics Engineers, Inc., New York, USA.
Dann, W. M., \& Montsinger, V. M. (1934). Overloading of power transformers. AIEE Transactions on Power Apparatus and Systems, 1353.

Fulczyk, M. (2001). Zero-sequence voltages in unit-connected generator for different methods of grounding generator neutral. Seventh International Conference on Developments in Power System Protection, IEE Conference Publication No. 479, p. 499-502.

García, F., \& Alcántara, R. (2006). $100 \%$ stator ground fault protection: a comparison of two protection methods. M.Sc. Thesis, Department of Industrial Electrical Engineering and Automation, Lund Institute of Technology, Sweden. www.iea.1th.se/publications/M S Theses/Full\%20document/5223_100\%25stator groundfaultprotection.pdf

Giraldo, L. C., Mora, J., \& Olguín, G. (2006). Análisis de sensitividad del método de inyección subarmónica de protección del $100 \%$ del estátor de un generador ante fallas a tierra. Segundo Congreso Internacional sobre Uso Racional y Eficiente de la Energía, Cali, Colombia.

IEEE (Institute of Electrical and Electronics Engineers). (1985). Standard C37.101. IEEE Guide for generator ground protection. The Institute of Electrical and Electronics Engineers, Inc., New York, USA.

IEEE (Institute of Electrical and Electronics Engineers). (1995). Standard C37.102. IEEE Guide for AC generator protection. The Institute of Electrical and Electronics Engineers, Inc., New York, USA.

Illar, M., Narayan, V., von Roeschlaub, F., \& Pencinger, C. J. (1979). Total generator ground fault protection. Protective Relaying Conference, Georgia Institute of Technology, Atlanta,Georgia, p. $70-75$. 
Powell, L. J. (1998). The impact of system grounding practices on generator fault damage. IEEE Transactions on Industry Applications 34 (5), 923-927.

Reimert, D. (2006). Protective relaying for power generation systems. CRC Press, Taylor and Francis Group, Boca Raton, Florida.

Wu, J., Wan, H., \& Lu, Y. (2002). Study of a fresh subharmonic injection scheme based on equilibrium principle for hydro-generator stator ground protection. IEEE Power Engineering Society Winter Meeting 2002, Vol. 2, p. 924-929. 